\title{
Effect of boundaries on the dynamic interaction of a liquid-filled porous layer and a supporting continuum
}

\author{
M TAJUDDIN and G NARAYAN REDDY \\ Department of Mathematics, Osmania University, Hyderabad 500 007, India, \\ e-mail: taj_osmania@yahoo.co.in
}

MS received 25 August 2004; revised 7 March 2005

\begin{abstract}
A transcendental equation is derived relating wave number and phase velocity of propagation of waves in a partially saturated layered half-space under plane strain conditions. The period equation is derived and discussed for two types of boundaries, permeable and impermeable. In the limiting cases, the problem reduces to more simplified forms as discussed by earlier researchers.
\end{abstract}

Keywords. Biot's theory; Rayleigh waves; Stoneley waves.

\section{Introduction}

Wave propagation in layered media is of great interest in seismology and geophysics, and a substantial amount of literature on this subject is available. An excellent survey of current knowledge in this field may be found in the treatise by Ewing et al (1957). A review of elastic wave propagation is given by Pao (1983). Free waves in the system of layer and half-space are investigated by Achenbach \& Epstein (1967).

In what follows, a study of effect of boundaries on the dynamic interaction of a liquid-filled porous layer of finite thickness $(h)$ resting on an elastic continuum is made, following Biot (1956). This model consists of an elastic matrix permeated by a network of interconnected spaces saturated with liquid. An account of further researches based on this model is available in Paria (1963) and a historical formulation by de Boer \& Ehlers (1988). Tajuddin (1984) and Tajuddin \& Lingam (1990) examined Rayleigh and Stoneley waves in poroelastic half-space and a porous solid lying over an elastic solid.

When both the layer and half-space are homogeneous and isotropic, the period equation is derived and discussed both for permeable as well as impermeable surfaces. Dispersive period equations are discussed in the limiting cases of wave number, and all interesting results are noted. Results with purely elastic solid are shown as a special case.

The investigation is of interest in earthquake engineering applications, involving dynamics of layered soil deposits of uniform thickness in which the ground water table originates at a certain depth below the surface. 


\section{Solution of the problem}

The coordinate system for the said problem under consideration is such that waves propagate in the $x$-direction, with amplitude decay in the $z$-direction taking the origin at the free surface. Let the two media be in contact along the plane $z=0$ such that $z>0$ defines the lower half-space. The solid displacement functions, $\mathbf{u}(u, w)$ that can readily be evaluated from the equations of motion of Biot (1956) representing time harmonic waves travelling in the $x$ direction (Tajuddin and Reddy 2004) are,

$$
\begin{aligned}
u= & -i k\left[C_{1} e^{p z}+C_{2} e^{-p z}+D_{1} e^{q z}+D_{2} e^{-q z}-i r_{0} A_{1} e^{r z}+i r_{0} B_{1} e^{-r z}\right] e^{i(\omega t-k x)}, \\
w= & k\left[C_{1} p_{0} e^{p z}-C_{2} p_{0} e^{-p z}+D_{1} q_{0} e^{q z}-D_{2} q_{0} e^{-q z}\right. \\
& \left.-i A_{1} e^{r z}-i B_{1} e^{-r z}\right] e^{i(\omega t-k x)}
\end{aligned}
$$

where $k$ is the wave number and $\omega$ is the frequency of the wave. $C_{1}, C_{2}, D_{1}, D_{2}, A_{1}, B_{1}$ are all constants and $p, q, r$ are

$$
\begin{aligned}
& p_{0}=p k^{-1}=\left(1-C^{2} C_{f d}^{-2}\right)^{1 / 2}, q_{0}=q k^{-1}=\left(1-C^{2} C_{s d}^{-2}\right)^{1 / 2} ; \\
& r_{0}=r k^{-1}=\left(1-C^{2} C_{s}^{-2}\right)^{1 / 2},
\end{aligned}
$$

with $C=\omega / k, C_{f d}, C_{s d}, C_{s}$ are the dilatational wave velocities of the first and second kinds and shear wave velocity respectively. Substitution of (1) into the stress-displacement relations of Biot (1956) gives the relevant stresses to be

$$
\begin{aligned}
\sigma_{z z}= & k^{2}\left[\left\{2 N p_{0}^{2}-C^{2} C_{f d}^{-2}\left(A+Q \xi_{0}^{2}\right)\right\}\left(C_{1} e^{p z}+C_{2} e^{-p z}\right)\right. \\
& +\left\{2 N q_{0}^{2}-C^{2} C_{s d}^{-2}\left(A+Q \delta_{0}^{2}\right)\right\}\left(D_{1} e^{q z}+D_{2} e^{-q z}\right) \\
& \left.-2 N i r_{0}\left(A_{1} e^{r z}-B_{1} e^{-r z}\right)\right] e^{i(\omega t-k x)}, \\
\sigma_{z x}= & -i k^{2} N\left[2 p_{0}\left(C_{1} e^{p z}-C_{2} e^{-p z}\right)+2 q_{0}\left(D_{1} e^{q z}-D_{2} e^{-q z}\right)\right. \\
& \left.-i\left(1+r_{0}^{2}\right)\left(A_{1} e^{r z}+B_{1} e^{-r z}\right)\right] e^{i(\omega t-k x)}, \\
s= & -C^{2} k^{2}\left[C_{f d}^{-2}\left(Q+R \xi_{0}^{2}\right)\left(C_{1} e^{p z}+C_{2} e^{-p z}\right)\right. \\
& \left.+C_{s d}^{-2}\left(Q+\delta_{0}^{2} R\right)\left(D_{1} e^{q z}+D_{2} e^{-q z}\right)\right] e^{i(\omega t-k x)},
\end{aligned}
$$

where $A, N, Q, R$ are all poroelastic constants and $\rho_{i j}$ are mass coefficients following Biot (1956) and

$$
\begin{aligned}
\xi_{0}^{2} & =\frac{P R-Q^{2}}{c_{f d}^{2}\left(R M_{12}-Q M_{22}\right)}-\frac{R M_{11}-Q M_{12}}{R M_{12}-Q M_{22}}, \\
M_{11} & =\rho_{11}-i b \omega^{-1}, M_{12}=\rho_{12}+i b \omega^{-1}, M_{22}=\rho_{22}-i b \omega^{-1}, P=A+2 N, \\
\delta_{0}^{2} & =\text { similar expression to } \xi_{0}^{2} \text { with } C_{\mathrm{fd}} \text { replaced by } C_{\text {sd }} \text { respectively. }
\end{aligned}
$$

Now, we shall consider the motion in the elastic half-space following its field equations. Further, the displacements and stresses must decrease with increasing distances form the 
plane $z=0$ and the subscript 1 is used for dependent variables and material of parameters. Proceeding as before, the displacement and stress functions are

$$
\begin{aligned}
u_{1} & =-i k\left[A_{3} e^{-\eta z}+i \zeta_{1} B_{3} e^{-\zeta z}\right] e^{i(\omega t-k x)}, \\
w_{1} & =-k\left(\eta_{1} A_{3} e^{-\eta z}+i B_{3} e^{-\zeta z}\right) e^{i(\omega t-k x)}, \\
\left(\sigma_{z z}\right)_{1} & =\mu_{1} k^{2}\left[\left(1+\zeta_{1}^{2}\right) A_{3} e^{-\eta z}+2 i \zeta_{1} B_{3} e^{-\zeta z}\right] e^{i(\omega t-k x)}, \\
\left(\sigma_{z x}\right)_{1} & =\mu_{1} k^{2} i\left[2 \eta_{1} A_{3} e^{-\eta z}+i\left(1+\zeta_{1}^{2}\right) B_{3} e^{-\zeta z}\right] e^{i(\omega t-k x),}
\end{aligned}
$$

where $A_{3}, B_{3}$ are constants and $\eta, \zeta$ are

$$
\eta_{1}=\eta k^{-1}=\left(1-C^{2} \alpha_{1}^{-2}\right)^{1 / 2}, \zeta_{1}=\zeta k^{-1}=\left(1-C^{2} \beta_{1}^{-2}\right)^{1 / 2},
$$

with $\mu_{1}$ shear modulus of the medium, and $\alpha_{1}, \beta_{1}$ the dilatational and shear wave velocity respectively.

\section{Boundary conditions - Frequency equation}

A non-dissipative medium is considered for welded contact both for permeable and impermeable surfaces, when the phase velocity $c$ is simply $\omega \mathrm{k}^{-1}$. For a permeable boundary, the liquid pressures in the pores vanish, while for an impermeable boundary the liquid pressure in the direction normal to the boundary vanishes. In this case, the boundary conditions are

$$
\begin{array}{ll}
\text { at } z=0, & \sigma_{z z}+s=0, \\
& \sigma_{z x}=0, \\
& s=0, \\
& \partial s / \partial z=0, \\
\text { at } z=h, \quad & \sigma_{z z}+s=\left(\sigma_{z z}\right)_{1}, \\
& \sigma_{z x}=\left(\sigma_{z x}\right)_{1}, \\
& u=u_{1}, \\
& w=w_{1}, \\
& s=0, \\
& \partial s / \partial z=0,
\end{array}
$$

where the subscript 1 relates to the half-space, while stresses and displacements without the subscript relate to the layer. Equations 5(a), 5(b), 5(d) and 5(e) have to be satisfied for a permeable surface, while 5(a), 5(c), 5(d) and 5(f) have to be satisfied for an impermeable surface. Equations (1), (3), (4) with the boundary conditions for permeable surfaces give a set of eight homogeneous equations for the eight constants $C_{1}, C_{2}, D_{1}, D_{2}, A_{1}, B_{1}, A_{3}, B_{3}$. In addition, we analyse further by introducing dimensionless variables given as under:

$$
\begin{aligned}
& a_{1}=P / H, a_{2}=Q / H, a_{3}=R / H, a_{4}=N / H, \\
& d_{1}=\rho_{11} / \rho, d_{2}=\rho_{12} / \rho, d_{3}=\rho_{22} / \rho, d_{4}=\rho_{1} / \rho, \\
& b_{1}=\mu_{1} / H, b_{2}=\lambda_{1} / H, \tilde{x}=\left(v_{o} / C_{f d}\right)^{2}, \tilde{y}=\left(v_{o} / C_{s d}\right)^{2}, \tilde{z}=\left(v_{o} / C_{s}\right)^{2} .
\end{aligned}
$$


Also, $m$ is dimensionless phase velocity, and

$$
\begin{gathered}
V_{o}^{2}=H / \rho, m=C / C_{o}, C=\omega k^{-1}, C_{o}^{2}=\mu_{1} / \rho_{1}, \\
\rho=\rho_{11}+2 \rho_{12}+\rho_{22}, H=P+2 Q+R .
\end{gathered}
$$

Then this system of homogeneous equations for a permeable surface can be represented in matrix form

$$
[\Delta][X]=0,
$$

in which

$$
\begin{aligned}
& {\left[X^{T}\right]=\left\{X_{1}, X_{2}\right\},} \\
& {\left[X_{1}^{T}\right]=\left\{C_{1} e^{p_{o}^{*} \theta}, D_{1} e^{q_{o}^{*} \theta},-i A_{1} e^{r_{o}^{*} \theta},\right\}} \\
& {\left[X_{2}^{T}\right]=\left\{C_{2} e^{-p_{o}^{*} \theta}, D_{2} e^{-q_{o}^{*} \theta}, i B_{1} e^{-r_{o}^{*} \theta}, A_{3} e^{-\eta_{1}^{*} \theta}, i B_{3} e^{-\zeta_{1}^{*} \theta}\right\},}
\end{aligned}
$$

and, therefore, for a non-trivial solution to exist, the frequency equation is

$$
|\Delta|=\left|\begin{array}{ll}
\Delta_{11} & \Delta_{12} \\
\Delta_{21} & \Delta_{22}
\end{array}\right|=0
$$

where the dimensionless wave number is $\theta(=k h)$, and

$$
\begin{aligned}
& {\left[\Delta_{11}\right]=\left[\begin{array}{ccc}
A_{11} e^{-p_{o}^{*} \theta} & A_{12} e^{-q_{o}^{*} \theta} & A_{13} e^{-r_{o}^{*} \theta} \\
A_{21} e^{-p_{o}^{*} \theta} & A_{22} e^{-q_{o}^{*} \theta} & A_{23} e^{-r_{o}^{*} \theta} \\
A_{31} e^{-p_{o}^{*} \theta} & A_{32} e^{-q_{o}^{*} \theta} & 0
\end{array}\right]} \\
& {\left[\Delta_{12}\right]=\left[\begin{array}{ccccc}
A_{11} e^{p_{o}^{*} \theta} & A_{12} e^{q_{o}^{*} \theta} & A_{13} e^{r_{o}^{*} \theta} & 0 & 0 \\
-A_{21} e^{p_{o}^{*} \theta} & -A_{22} e^{q_{o}^{*} \theta} & -A_{23} e^{r_{o}^{*} \theta} & 0 & 0 \\
A_{31} e^{p_{o}^{*} \theta} & A_{32} e^{q_{o}^{*} \theta} & 0 & 0 & 0
\end{array}\right] \text {, }} \\
& {\left[\Delta_{21}^{T}\right]=\left[\begin{array}{ccccc}
A_{11} & a_{4} b_{1}^{-1} A_{21} & 1 & p_{o}^{*} & A_{31} \\
A_{12} & a_{4} b_{1}^{-1} A_{22} & 1 & q_{o}^{*} & A_{32} \\
A_{13} & a_{4} b_{1}^{-1} A_{23} & r_{o}^{*} & 1 & 0
\end{array}\right]} \\
& {\left[\Delta_{22}\right]=\left[\begin{array}{ccccc}
A_{11} & A_{12} & A_{13} & -A_{41} & -A_{42} \\
-a_{4} b_{1}^{-1} A_{21} & -a_{4} b_{1}^{-1} A_{22} & -a_{4} b_{1}^{-1} A_{23} & A_{51} & A_{41} \\
1 & 1 & r_{o}^{*} & -\xi_{1}^{*} & -\zeta_{1}^{*} \\
-p_{o}^{*} & -q_{o}^{*} & -1 & \eta_{1}^{*} & 1 \\
A_{31} & A_{32} & 0 & 0 & 0
\end{array}\right] .}
\end{aligned}
$$

In (7), the elements are

$$
\begin{aligned}
& A_{11}=2 a_{4} b_{1}^{-1} p_{0}^{*^{2}}-m^{2} \tilde{x} d_{4}^{-1}\left\{\left(a_{1}-2 a_{4}+a_{2}\right)+\left(a_{2}+a_{3}\right) \xi_{0}^{*^{2}}\right\}, \\
& A_{13}=2 a_{4} b_{1}^{-1} r_{0}^{*}, A_{21}=2 p_{0}^{*}, A_{22}=2 q_{0}^{*}, A_{23}=1+r_{0}^{*^{2}}, \\
& A_{31}=-m^{2} \tilde{x} d_{4}^{-1}\left(a_{2}+a_{3} \xi_{0}^{*^{2}}\right), A_{41}=2-m^{2}, A_{42}=2\left(1-m^{2}\right)^{1 / 2}, \\
& A_{51}=2\left[1-m^{2} b_{1}\left(2 b_{1}+b_{2}\right)^{-1}\right]^{1 / 2},
\end{aligned}
$$


$A_{12}, A_{32}=$ similar expressions to $A_{11}, A_{31}$, with $p_{0}^{*^{2}}, \xi_{0}^{*^{2}}$ replaced by $q_{0}^{*^{2}}, \delta_{0}^{*^{2}}$ respectively. In writing the above elements, we also have

$$
\begin{aligned}
p_{0}^{*} & =\left(1-m^{2} \tilde{x} b_{1} d_{4}^{-1}\right)^{1 / 2}, q_{0}^{*}=\left(1-m^{2} \tilde{y} b_{1} d_{4}^{-1}\right)^{1 / 2}, \\
r_{0}^{*} & =\left(1-m^{2} \tilde{z} b_{1} d_{4}^{-1}\right)^{1 / 2}, \xi_{1}^{*}=\left(1-m^{2}\right)^{1 / 2}, 2 \eta_{1}^{*}=A_{51}, \\
\xi_{0}^{*^{2}} & =\frac{\tilde{x}\left(a_{1} a_{3}-a_{2}^{2}\right)}{a_{3} d_{2}-a_{2} d_{3}}-\frac{a_{3} d_{1}-a_{2} d_{2}}{a_{3} d_{2}-a_{2} d_{3}}, \\
\delta_{0}^{*^{2}} & =\text { similar expression to } \xi_{0}^{*^{2}} \text { with } \tilde{x} \text { replaced by } \tilde{y}, \text { respectively. }
\end{aligned}
$$

In view of (7), the phase velocity and wave number are related through a transcendental equation and are expected to have complex roots. We also note that the submatrices $\left[\Delta_{11}\right],\left[\Delta_{12}\right],\left[\Delta_{21}\right]$ in (7) are purely dependent on the properties of the porous layer, while $\left[\Delta_{22}\right]$, in addition, depends on the properties of the underlying half-space. If the matrix $\left[\Delta_{22}\right]$ is singular, the results for a permeable surface in case of welded contact obtained by Tajuddin \& Lingam (1990) are recovered as a special case. If the layer is also assumed to be of a dry type, then the results due to Achenbach \& Epstein (1967) are deduced as a particular case. Arguing on similar lines, the frequency equation for an impermeable surface can also be written.

\section{Special cases}

Now, we examine the cases that involve the limits of wave number $\theta(=k h)$. For this purpose, consider the solution of the $2 \times 2$ partitioned system in (7) for a permeable surface, i.e.,

$$
\left[\Delta_{21}\right]\left[\Delta_{11}^{-1}\right]\left[\Delta_{12}\right]+\left[\Delta_{22}\right]=0,
$$

which after performing the necessary computations takes the form,

$$
\left\{[D]+R_{L}(k)\left[\Delta_{22}\right]\right\}=0,
$$

where

$$
R_{L}(k)=A_{31}\left(A_{12} A_{23}-A_{22} A_{13}\right)-A_{32}\left(A_{11} A_{23}-A_{21} A_{13}\right),
$$

and $\left|D_{i j}\right|=0, i, j=1,2, \ldots \ldots \ldots, 5$,

$$
\begin{aligned}
& D_{11}=A_{11} X_{11} e^{-\left(q_{o}^{*}+r_{o}^{*}-p_{o}^{*}\right) \theta}+A_{12} X_{12} e^{-r_{o}^{*} \theta}+A_{13} X_{13} e^{-q_{o}^{*} \theta}, \\
& D_{12}=A_{11} Y_{11} e^{-r_{o}^{*} \theta}+A_{12} Y_{12} e^{-\left(p_{o}^{*}+r_{o}^{*}-q_{o}^{*}\right) \theta}+A_{13} Y_{13} e^{-p_{o}^{*} \theta}, \\
& D_{13}=A_{11} Z_{11} e^{-q_{o}^{*} \theta}+A_{12} Z_{12} e^{-p_{o}^{*} \theta}+A_{13} Z_{13} e^{-\left(p_{o}^{*}+q_{o}^{*}-r_{o}^{*}\right) \theta}, \\
& D_{21}=a_{4} b_{1}^{-1}\left(A_{21} X_{11} e^{-\left(q_{o}^{*}+r_{o}^{*}-p_{o}^{*}\right) \theta}+A_{22} X_{12} e^{-r_{o}^{*} \theta}+A_{23} X_{13} e^{-q_{o}^{*} \theta}\right), \\
& D_{22}=a_{4} b_{1}^{-1}\left(A_{21} Y_{11} e^{-r_{o}^{*} \theta}+A_{22} Y_{12} e^{-\left(p_{o}^{*}+r_{o}^{*}-q_{o}^{*}\right) \theta}+A_{23} Y_{13} e^{-p_{o}^{*} \theta}\right), \\
& D_{23}=a_{4} b_{1}^{-1}\left(A_{21} Z_{11} e^{-q_{o}^{*} \theta}+A_{22} Z_{12} e^{-p_{o}^{*} \theta}+A_{23} Z_{13} e^{-\left(p_{o}^{*}+q_{o}^{*}-r_{o}^{*}\right) \theta}\right), \\
& D_{31}=X_{11} e^{-\left(q_{o}^{*}+r_{o}^{*}-p_{o}^{*}\right) \theta}+X_{12} e^{-r_{o}^{*} \theta}+r_{0}^{*} X_{13} e^{-q_{o}^{*} \theta}, \\
& D_{32}=Y_{11} e^{-r_{o}^{*} \theta}+Y_{12} e^{-\left(p_{o}^{*}+r_{o}^{*}-q_{o}^{*}\right) \theta}+r_{0}^{*} Y_{13} e^{-p_{o}^{*} \theta},
\end{aligned}
$$




$$
\begin{aligned}
& D_{33}=Z_{11} e^{-q_{o}^{*} \theta}+Z_{12} e^{-p_{o}^{*} \theta}+r_{0}^{*} Z_{13} e^{-\left(p_{o}^{*}+q_{o}^{*}-r_{o}^{*}\right) \theta}, \\
& D_{41}=p_{0}^{*} X_{11} e^{-\left(q_{o}^{*}+r_{o}^{*}-p_{o}^{*}\right) \theta}+q_{0}^{*} X_{12} e^{-r_{o}^{*} \theta}+X_{13} e^{-q_{o}^{*} \theta}, \\
& D_{42}=p_{0}^{*} Y_{11} e^{-r_{o}^{*} \theta}+q_{0}^{*} Y_{12} e^{-\left(p_{o}^{*}+r_{o}^{*}-q_{o}^{*}\right) \theta}+Y_{13} e^{-p_{o}^{*} \theta}, \\
& D_{43}=p_{0}^{*} Z_{11} e^{-q_{o}^{*} \theta}+q_{0}^{*} Z_{12} e^{-p_{o}^{*} \theta}+Z_{13} e^{-\left(p_{o}^{*}+q_{o}^{*}-r_{o}^{*}\right) \theta}, \\
& D_{51}=A_{31} X_{11} e^{-\left(q_{o}^{*}+r_{o}^{*}-p_{o}^{*}\right) \theta}+A_{32} X_{12} e^{-r_{o}^{*} \theta}, \\
& D_{52}=A_{31} Y_{11} e^{-r_{o}^{*} \theta}+A_{32} Y_{12} e^{-\left(p_{o}^{*}+r_{o}^{*}-q_{o}^{*}\right) \theta}, \\
& D_{53}=A_{31} Z_{11} e^{-q_{o}^{*} \theta}+A_{32} Z_{12} e^{-p_{o}^{*} \theta}, \\
& D_{14}=D_{15}=D_{24}=D_{25}=D_{34}=D_{35}=D_{44}=D_{45}=D_{54}=D_{55}=0,
\end{aligned}
$$

with

$$
\begin{aligned}
& X_{11}=A_{31}\left(A_{12} A_{23}-A_{13} A_{22}\right)-A_{32}\left(A_{11} A_{23}-A_{13} A_{21}\right), \\
& X_{12}=2 A_{13} A_{21} A_{31}, X_{13}=2 A_{21}\left(A_{11} A_{32}-A_{12} A_{31}\right), \\
& Y_{11}=-2 A_{13} A_{22} A_{32}, Y_{12}=A_{31}\left(A_{12} A_{23}+A_{13} A_{22}\right)+A_{32}\left(A_{13} A_{21}-A_{11} A_{23}\right), \\
& Y_{13}=2 A_{22}\left(A_{11} A_{32}-A_{12} A_{31}\right), Z_{11}=-2 A_{13} A_{23} A_{32}, Z_{12}=2 A_{13} A_{23} A_{31}, \\
& Z_{13}=A_{32}\left(A_{13} A_{21}+A_{11} A_{23}\right)-A_{31}\left(A_{13} A_{22}+A_{12} A_{23}\right) .
\end{aligned}
$$

Since the phase velocity depends on the wave number, the harmonic waves are dispersive. It can be inferred from a poroelastic deformable solid that

$$
p_{0}^{*}>0, q_{0}^{*}>0, r_{0}^{*}>0,
$$

which also implies

$$
\left(p_{0}^{*}+r_{0}^{*}\right)>q_{0}^{*},\left(p_{0}^{*}+q_{0}^{*}\right)>r_{0}^{*},\left(q_{0}^{*}+r_{0}^{*}\right)>p_{0}^{*} .
$$

Thus, we see that the exponential terms in (11) are certainly negative multiples of dimensionless wave number, $\theta$. By increasing the thickness of layer $h$ while keeping the wave number fixed, then at the limit $\theta \rightarrow \infty$, each exponential term in (11) tends to zero and consequently the matrix $[D]$ in (9) approaches zero, and thus the frequency equation reduces to

$$
R_{L}(k)\left[\Delta_{22}\right]=0,
$$

Hence, at the short wavelength limit, the phase velocity approaches the velocity of Rayleigh waves in the layer $\left(R_{L}(k)=0\right)$ or the velocity of Stoneley waves at the interface between a porous layer lying over an elastic half-space $\left(\left|\Delta_{22}\right|=0\right)$, if such waves can exist (similar to the problems considered by Tajuddin 1984 and Tajuddin \& Lingam 1990).

Similarly, by keeping the wave number constant and letting the thickness of the layer approach zero, (9) reduces to the frequency equation for Rayleigh waves associated with an elastic half-space. Hence, for very thin layers, the phase velocity approaches the velocity of Rayleigh waves in the underlying half-space.

In case of an impermeable surface, proceeding on similar lines and arguing as before, one obtains

$$
\left\{[\tilde{D}]+\tilde{R}_{L}(k)\left[\tilde{\Delta}_{22}\right]\right\}=0,
$$


where

$$
\begin{aligned}
\tilde{R}_{L}(k)= & \text { similar expression as } R_{L}(k) \text { with } A_{31}, A_{32} \text { replaced by } \\
& p_{0}^{*} A_{31}, q_{0}^{*} A_{32}, \text { respectively, } \\
{\left[\tilde{\Delta}_{22}\right]=} & \text { similar matrix as }\left[\Delta_{22}\right] \text { with } A_{31}, A_{32} \text { replaced by } \\
& -p_{0}^{*} A_{31},-q_{0}^{*} A_{32}, \text { respectively, }
\end{aligned}
$$

and $\left|\tilde{D}_{i j}\right|=0, i, j=1,2, \ldots, 5$,

$$
\begin{aligned}
\tilde{D}_{11}, \tilde{D}_{21}, \tilde{D}_{31}, \tilde{D}_{41}= & \text { similar expressions to } D_{11}, D_{21}, D_{31}, D_{41} \text { with } \\
& X_{11}, X_{12}, X_{13} \text { replaced by } \tilde{X}_{11}, \tilde{X}_{12}, \tilde{X}_{13} \text {, respectively, }
\end{aligned}
$$

$\tilde{D}_{12}, \tilde{D}_{22}, \tilde{D}_{32}, \tilde{D}_{42}=$ similar expressions to $D_{12}, D_{22}, D_{32}, D_{42}$ with $Y_{11}, Y_{12}, Y_{13}$ replaced by $\tilde{Y}_{11}, \tilde{Y}_{12}, \tilde{Y}_{13}$, respectively,

$\tilde{D}_{13}, \tilde{D}_{23}, \tilde{D}_{33}, \tilde{D}_{43}=$ similar expressions to $D_{13}, D_{23}, D_{33}, D_{43}$ with

$$
Z_{11}, Z_{12}, Z_{13} \text { replaced by } \tilde{Z}_{11}, \tilde{Z}_{12}, \tilde{Z}_{13} \text {, respectively, }
$$

$\tilde{D}_{51}, \tilde{D}_{52}, \tilde{D}_{53}=$ similar expressions to $D_{51}, D_{52}, D_{53}$, with

$X_{11}, X_{12} ; Y_{11}, Y_{12} ; Z_{11}, Z_{12}$ and $A_{31}, A_{32}$ replaced by

$\tilde{X}_{11}, \tilde{X}_{12} ; \tilde{Y}_{11}, \tilde{Y}_{12} ; \tilde{Z}_{11}, \tilde{Z}_{12}$ and $-p_{0}^{*} A_{31},-q_{0}^{*} A_{32}$,

respectively,

$$
\tilde{D}_{14}=\tilde{D}_{15}=\tilde{D}_{24}=\tilde{D}_{25}=\tilde{D}_{34}=\tilde{D}_{35}=\tilde{D}_{44}=\tilde{D}_{45}=\tilde{D}_{54}=\tilde{D}_{55}=0
$$

with

$$
\begin{aligned}
& \tilde{X}_{11}=p_{0}^{*} A_{31}\left(A_{13} A_{22}-A_{12} A_{23}\right)-q_{0}^{*} A_{32}\left(A_{11} A_{23}-A_{13} A_{21}\right), \\
& \tilde{X}_{12}=2 p_{0}^{*} A_{11} A_{23} A_{31}, \\
& \tilde{X}_{13}=2 A_{11}\left(q_{0}^{*} A_{21} A_{32}-p_{0}^{*} A_{22} A_{31}\right), \tilde{Y}_{11}=-2 p_{0}^{*} A_{12} A_{23} A_{32}, \\
& \tilde{Y}_{13}=2 A_{12}\left(q_{0}^{*} A_{21} A_{32}-p_{0}^{*} A_{22} A_{31}\right), \\
& \tilde{Y}_{12}=p_{0}^{*} A_{31}\left(A_{12} A_{23}+A_{13} A_{22}\right)-q_{0}^{*} A_{32}\left(A_{13} A_{21}-A_{11} A_{23}\right), \\
& \tilde{Z}_{11}=-2 q_{0}^{*} A_{13} A_{23} A_{32}, \tilde{Z}_{12}=2 p_{0}^{*} A_{13} A_{23} A_{31}, \\
& \tilde{Z}_{13}=q_{0}^{*} A_{32}\left(A_{13} A_{21}+A_{11} A_{23}\right)-p_{0}^{*} A_{31}\left(A_{13} A_{22}+A_{12} A_{23}\right) .
\end{aligned}
$$

At the short wavelength limit and for very thin layers, analogous conclusions like that of the permeable surface are obtained. By ignoring the liquid effects of pores, the results of Achenbeach \& Epstein (1967) are obtained as a special case. It is also interesting to note that for a smooth contact, irrespective of the wavelength limit, the phase velocity approaches the velocity of Rayleigh waves in the layer or the velocity of Stoneley waves at the interface between a porous elastic layer lying over an elastic half-space (Tajuddin \& Reddy 2004). 


\section{Conclusion}

The propagation of free waves in a partially saturated layered half-space has been examined for two types of boundaries, viz. permeable and impermeable. A set of poroelastic materials considered by Biot (1956) is chosen to compute phase velocity as a function of wave number and illustrated graphically. The material parameters are presented in appendix A. The investigation has led to the following conclusions.

(i) Harmonic waves, in general, are dispersive since phase velocity depends on wave number.

(ii) Velocity of shear waves in a half-space is an upper bound for phase velocities of free waves in a layer and a half-space.

(iii) At the short wavelength limit, phase velocity is the analogous Rayleigh equation for the layer or the analogous equation for Stoneley waves, each for permeable and impermeable surfaces.

(iv) In case of very thin layers, the phase velocity is the analogous Rayleigh equation of supporting half-space.

(v) If the velocity of shear waves in the layer is larger than the velocity of shear waves in the half-space, there exists only one mode of propagation; otherwise, there are many modes.

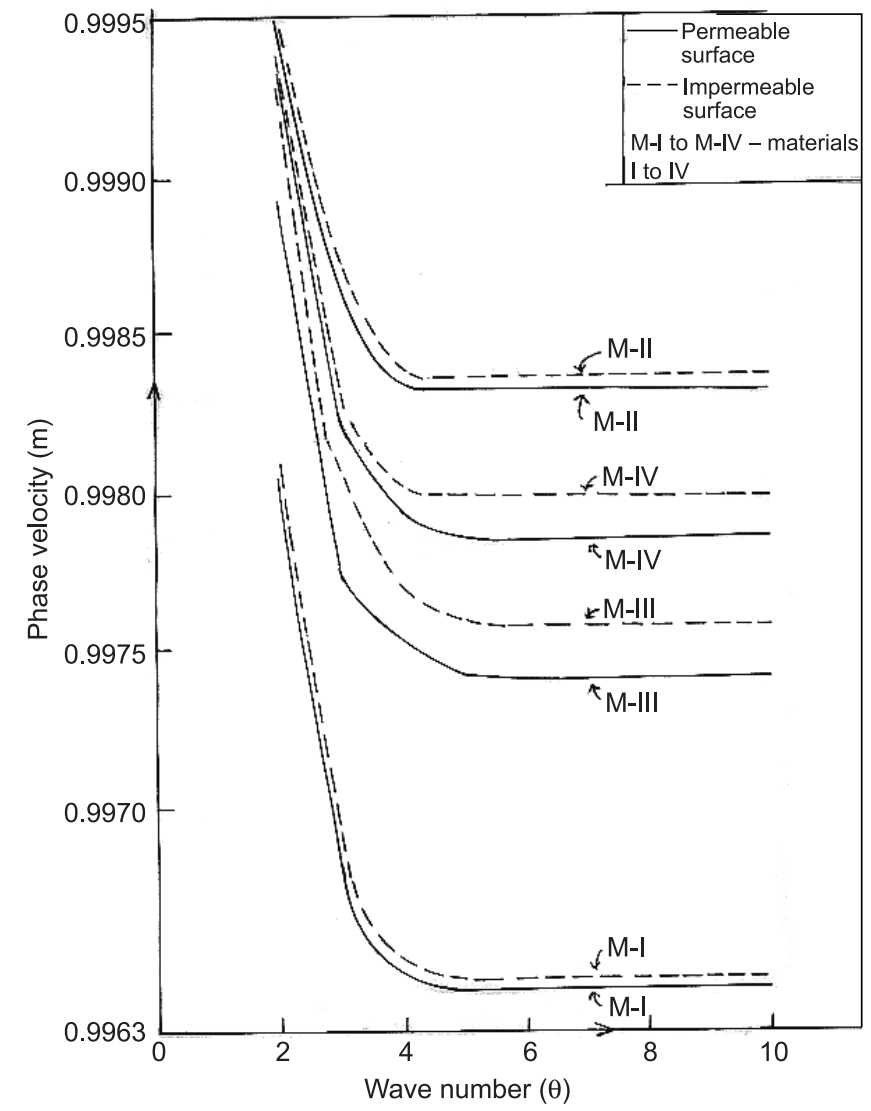

Figure 1. Phase velocity as a function of wave number. 
(vi) Figure 1 shows that the effect due to liquid is mainly associated with low wave number, and then remains constant uniformly.

(vii) Figure 1 shows that the effects of predominantly present mass of solid, elastic constants, mass-coupling parameter play a role in the phase velocity equation in a sequential manner.

(viii) Figure 1 shows that the phase velocity for an impermeable surface is higher than that of a permeable surface.

(ix) It is ascertained that the phase velocity is higher for a welded contact than for a smooth contact.

\section{Appendix A.}

Parameters of materials I to IV as given by Biot (1956) are shown in table A1.

Table A1. Parameters of materials I to IV (Biot 1956).

\begin{tabular}{lccccccccccccc}
\hline Material & $a_{1}$ & $a_{2}$ & $a_{3}$ & $a_{4}$ & $d_{1}$ & $d_{2}$ & $d_{3}$ & $d_{4}$ & $\tilde{x}$ & $\tilde{y}$ & $\tilde{z}$ & $b_{1}$ & $b_{2}$ \\
\hline I & 0.61 & 0.043 & 0.305 & 0.2066 & 0.5 & 0 & 0.5 & 1.1 & 0.812 & 1.674 & 1.1317 & 0.256 & 0.724 \\
II & 0.61 & 0.043 & 0.305 & 0.2066 & 0.8 & 0 & 0.2 & 1.1 & 0.65 & 1.339 & 0.7972 & 0.256 & 0.724 \\
III & 0.61 & 0.043 & 0.305 & 0.2066 & 0.65 & -0.15 & 0.65 & 1.1 & 0.909 & 2.394 & 1.0101 & 0.256 & 0.724 \\
IV & 0.74 & 0.037 & 0.185 & 0.2506 & 0.5 & 0 & 0.5 & 1.2 & 0.672 & 2.736 & 0.7182 & 0.287 & 0.872 \\
\hline
\end{tabular}

\section{References}

Achenbach J D, Epstein H I 1967 Dynamic interaction of a layer and a half-space. ASCE, J. Eng. Mech. 93(5): 27-42

Biot M A 1956 The theory of propagation of elastic waves in a fluid saturated porous solid. J. Acoust. Soc. Am. 28: 168-178

de Boer R, Ehlers W 1988 A historical review of the formulation of porous media theories. Acta Mech. 74: $1-8$

Ewing W M, Jardetzsky W S, Press F 1957 Elastic waves in layered media (New York: McGraw-Hill) Pao Y H 1983 Elastic waves in solids. ASME, J. Appl. Mech. E50: 1152-1164

Paria G 1963 Flow of fluid through porous deformable solids. Appl. Mech. Rev. 16: 421-423

Tajuddin M 1984 Rayleigh waves in a poroelastic half-space. J. Acoust. Soc. Am. 75: 682-684

Tajuddin M, Lingam P B 1990 Propagation of surface waves in a poroelastic solid layer lying over an elastic solid. Acta Geophys. Pol. 38: 279-287

Tajuddin M, Reddy G N 2004 Waves in partially saturated poroelastic layer resting on an elastic halfspace. Bull. Calutta Math. Soc. 96: 403-410 